\title{
Como aposentadorias e pensões afetam a educação e o trabalho de jovens do domicílio ${ }^{1}$
}

\author{
Rodolfo Hoffmann ${ }^{2}$
}

\section{Resumo}

A questão central é saber como o valor da parcela do rendimento domiciliar formada por aposentadorias e pensões afeta a probabilidade de jovens (15 a 21 anos de idade) desse domicílio não estarem estudando nem participando do mercado de trabalho. Combinando as possibilidades de estar ou não freqüentando escola e estar ou não no mercado de trabalho, há 4 situações, cujas probabilidades foram analisadas por meio de um lógite multinomial. Usando dados da PNAD de 2006 mostra-se que o rendimento de aposentadorias e pensões contribui claramente para aumentar a probabilidade de o jovem estudar e não participar do mercado de trabalho. Não há base para afirmar que aposentadorias e pensões contribuem para aumentar a probabilidade de que os jovens do domicílio não estudem nem participem do mercado de trabalho.

Palavra-chave: Aposentadorias; Jovens; Trabalho; Lógite mutinomial.

\section{Abstract \\ How pensions affect the schooling and labor of the household's youngsters}

The central question of this paper is to determine whether the component of the household income constituted by pensions affects the probability of the youngsters (15 to 21 years old) in the household being neither students nor labor suppliers. Combining the possibilities of attending or not attending school and participating or not in the labor market, there are 4 situations whose probabilities are analyzed using a multinomial logit model. Using data from the 2006 Brazilian National Household Sample Survey, it is shown that the income of pensioners contributes substantially to increase the probability of the household's youngsters being in school and not participating in the labor market. There is no evidence that pension incomes in a household increase the probability of their youngsters being simultaneously outside school and outside the labor market.

Key words: Pensions; Youngsters; Labor; Multinomial logit.

JEL C35, J22.

\section{Introdução}

Recente artigo de Reis e Camargo (2007) analisa os impactos de aposentadorias e pensões sobre a educação e a participação dos jovens na força de trabalho, usando dados da Pesquisa Nacional por Amostra de Domicílios (PNAD) de 2003. Analisando os dados dessa mesma PNAD e também os dados da PNAD de 2006 foi possível confirmar as

(1) Trabalho recebido em março de 2009 e aprovado em março de 2010. Este texto foi submetido para publicação em Pesquisa e Planejamento Econômico (PPE) em fevereiro de 2008. Em março de 2009 o autor recebeu comunicação de que o Corpo Editorial da revista tinha decidido publicar apenas a nota que saiu em PPE, v. 38, n. 3, p. 521.

(2) Professor do Instituto de Economia da Universidade Estadual de Campinas (IE/Unicamp), com apoio do CNPq (Conselho Nacional de Desenvolvimento Científico e Tecnológico), Campinas, SP, Brasil. E-mail: $<$ rhoffman@eco.unicamp.br>. 
principais conclusões desse artigo, excetuando-se a afirmativa de que aposentadorias e pensões levam a "um aumento na probabilidade de que os jovens não estejam estudando nem participando do mercado de trabalho". ${ }^{3}$ Tudo indica que se trata de um erro de interpretação dos coeficientes estimados de um modelo de lógite multinomial. A questão é relevante para a avaliação correta do efeito de aposentadorias e pensões nas decisões pessoais nas famílias e é interessante como exemplo das dificuldades de interpretação das estimativas dos parâmetros em tal tipo de modelo.

\section{0 modelo}

São considerados os jovens com idade de 15 a 21 anos, moradores de áreas urbanas, em domicílios com adultos com idade de 22 a 64 anos. ${ }^{4}$ Esses jovens são classificados em 4 categorias. Para simplificar a definição das 4 categorias, o termo "trabalha" será usado para indicar que a pessoa é economicamente ativa (trabalha ou procura trabalho) e o termo "estuda" será usado para indicar que o jovem frequienta uma escola. As 4 categorias exaustivas e mutuamente exclusivas são:

1 - Trabalha e não estuda

2 - Trabalha e estuda

3 - Estuda e não trabalha

4 - Não trabalha nem estuda.

Esses 4 eventos serão indicados por $E_{h}, \operatorname{com} h=1,2,3$ ou 4 . A probabilidade de o $i$-ésimo jovem pertencer à categoria $h$ é indicada por $P_{i h}$. Deseja-se analisar como a probabilidade $P_{i h}$ depende das características do jovem (incluindo sua idade, escolaridade, cor e sexo) e do seu domicílio (renda per capita, região, etc.).

Seja $\mathbf{x}_{i}^{\prime}$ o vetor-linha com os valores das variáveis explanatórias para o $i$-ésimo jovem e sejam $\boldsymbol{\beta}_{2}, \boldsymbol{\beta}_{3}$ e $\boldsymbol{\beta}_{4}$ os vetores-coluna de parâmetros para as categorias 2,3 e 4 , respectivamente. $\mathrm{O}$ modelo de lógite multinomial para esse problema pode ser expresso pelas seguintes equações ${ }^{6}$ :

(3) Deveria ser desnecessário dizer que a afirmativa não pode ser negada "por princípio". Em tese, transferências de renda podem, sim, favorecer a ociosidade de pessoas direta ou indiretamente beneficiadas. Para saber se o fenômeno ocorre ou não é indispensável a análise cuidadosa das informações disponíveis e/ou coleta de novos dados.

(4) No artigo de Reis e Camargo (2007) ocorre certa imprecisão na definição das variáveis. Faz-se referência a "jovens com idade entre 15 e 21 anos" quando o correto seria "jovens com idade de 15 a 21 anos". Na p. 225 define-se adultos como pessoas com mais de 21 anos, mas na tabela 2 consta que adultos são pessoas de 22 a 64 anos.

(5) É claro que a categoria poderia se limitar aos jovens ocupados, mas optou-se por manter a definição utilizada por Reis e Camargo (2007), cujo objetivo era analisar o impacto de aposentadorias e pensões na oferta de trabalho dos jovens, ou seja, na sua participação na força de trabalho.

(6) Nas equações (3) do artigo de Reis e Camargo, e também no parágrafo seguinte, aparece indevidamente um índice $j$ na representação do vetor das variáveis explanatórias. 


$$
P_{i 1}=\frac{1}{1+\sum_{j=2}^{4} \exp \left(\mathbf{x}_{i}^{\prime} \boldsymbol{\beta}_{j}\right)}
$$

e

$$
P_{i h}=\frac{\exp \left(\mathbf{x}_{i}^{\prime} \boldsymbol{\beta}_{h}\right)}{1+\sum_{j=2}^{4} \exp \left(\mathbf{x}_{i}^{\prime} \boldsymbol{\beta}_{j}\right)} \quad \text { para } h=2,3 \text { ou } 4
$$

Como se trata de 4 categorias exaustivas e mutuamente exclusivas, as 4 probabilidades para uma mesma pessoa sempre somam 1. Uma das 4 probabilidades pode ser obtida por diferença. Neste caso a categoria 1 (trabalha e não estuda) foi adotada como "base".

Greene (2000, p. 861) ressalta que é tentador associar o vetor de parâmetros $\boldsymbol{\beta}_{h}$ com o resultado $E_{h}$ (ou com sua probabilidade $P_{h}$ ), mas isso seria errôneo. Note-se, nas expressões (1) e (2), que as probabilidades $P_{i h}(\operatorname{com} h=1, \ldots, 4)$ dependem de todos os 3 vetores de parâmetros.

É fácil verificar, a partir de (1) e (2), que

$$
\frac{P_{i h}}{P_{i 1}}=\exp \left(\mathbf{x}_{i}^{\prime} \boldsymbol{\beta}_{h}\right) \quad \operatorname{para} h=2,3 \text { ou } 4
$$

De maneira mais geral, definindo $\boldsymbol{\beta}_{1}$ como um vetor-coluna de zeros, e indicando por $h$ e $k$ duas categorias quaisquer, tem-se

$$
\frac{P_{i h}}{P_{i k}}=\exp \left[\mathbf{x}_{i}^{\prime}\left(\boldsymbol{\beta}_{h}-\boldsymbol{\beta}_{k}\right)\right] \quad \text { para } h, k=1, \ldots, 4
$$

Os 4 eventos possíveis são obviamente dependentes entre si, pois se trata de 4 categorias mutuamente exclusivas. Mas, como mostra a expressão (4), no modelo de lógite multinomial a relação entre as probabilidades associadas às categorias $h$ e $k$ depende apenas dos vetores $\boldsymbol{\beta}_{h}$ e $\boldsymbol{\beta}_{k}$, não sendo afetada por uma redefinição das outras categorias.

Seja $X_{m i}$ uma determinada variável do vetor $\mathbf{x}_{i}^{\prime}$ e sejam $\beta_{2 m}, \beta_{3 m}$ e $\beta_{4 m}$ os respectivos parâmetros. Se, por exemplo, $\beta_{2 m}>0$, pode-se concluir, de acordo com (3), que a relação $P_{i 2} / P_{i 1}$ cresce em função de $X_{m i}$. Mas, havendo mais de duas categorias, isso não permite concluir que $P_{i 2}$ cresce em função de $X_{m i}$. É perfeitamente possível que a relação $P_{i 2} / P_{i 1}$ cresça com os valores de $P_{i 2}$ e $P_{i 1}$ se reduzindo, desde que a redução de $P_{i 1}$ seja proporcionalmente maior do que a redução de $P_{i 2}$.

No caso do lógite binomial, com apenas duas categorias, o crescimento da relação $P_{i 2} / P_{i 1}$ significa, obviamente, que $P_{i 2}$ cresce e $P_{i 1}$ diminui, já que $P_{i 1}+P_{i 2}=1$. 
Rodolfo Hoffmann

\section{Variáveis explanatórias e dados utilizados}

Segue-se a lista das variáveis explanatórias consideradas.

a) Idade do jovem e o quadrado dessa idade.

b) Sexo, assumindo valor 1 para feminino e 0 para masculino.

c) 4 variáveis binárias para cor ou raça (indígena, branco, preto, pardo e amarelo, adotando branco como base).

d) 3 variáveis binárias para distinguir 4 níveis de escolaridade (nula, 1 a 8 anos, 9 ou 10 e 11 anos ou mais), adotando como base a escolaridade nula. Também foram feitos ajustamentos utilizando 15 variáveis binárias para distinguir 16 níveis de escolaridade, sem que isso alterasse substancialmente os resultados.

e) 5 variáveis binárias para distinguir regiões do país, adotando o Nordeste como base e destacando SP do Sudeste.

f) Número de crianças com até 5 anos no domicílio.

g) Número de crianças com 6 a 10 anos no domicílio.

h) Número de moradores com 11 a 14 anos.

i) Número de jovens com 15 a 21 anos, excluindo o jovem cuja categoria é a variável dependente.

j) Rendimento domiciliar per capita "corrigido", excluindo do rendimento domiciliar os rendimentos de aposentadorias e pensões e o eventual rendimento de trabalho do jovem considerado na definição da variável dependente, em centenas de reais correntes.

k) Escolaridade média dos adultos (com 22 a 64 anos) do domicílio.

1) Uma variável binária que indica a presença de aposentadoria e/ou pensão no rendimento domiciliar ou, alternativamente, o valor per capita de aposentadorias e pensões, em centenas de reais correntes.

Verifica-se que foram utilizadas 22 variáveis explanatórias. Incluindo as constantes, o modelo de lógite multinomial, com 4 categorias, fica com $3 \times 23=69$ parâmetros.

Cabe ressaltar que na contagem do número de moradores do domicílio foram sempre excluídas as pessoas cuja condição no domicílio é de pensionista, empregado doméstico ou parente de empregado doméstico.

Foram considerados apenas domicílios particulares, excluindo todos os que tivessem valor não declarado para alguma variável relevante para a análise.

Todas as análises estatísticas foram feitas com ponderação pelo fator de expansão fornecido pelo IBGE nos microdados da PNAD.

Utilizando os dados da PNAD de 2003 (sem as variáveis do questionário suplementar), foram obtidos resultados qualitativamente equivalentes aos apresentados por Reis e Camargo (2007). As diferenças numéricas se devem, provavelmente, ao fato de não serem usados exatamente os mesmos filtros nos dados ou a uma eventual diferença na maneira de definir alguma variável a partir das informações disponíveis nos microdados. 

de 2006 .

Optou-se por apresentar aqui apenas alguns dos resultados obtidos com a PNAD

\section{Resultados para 2006}

Nos microdados da PNAD de 2006 são encontrados, na amostra, 40.539 jovens com idade de 15 a 21 anos, em domicílios particulares com pelo menos um adulto com idade de 22 a 64 anos, excluindo os domicílios onde houvesse pessoa com falta de informação sobre alguma das variáveis a serem utilizadas na análise (rendimento, escolaridade, idade, frequiência à escola, cor e ser ou não economicamente ativa). Esses 40.539 jovens estão em 29.961 domicílios, correspondendo, na população, a 18.174.353 jovens em 13.553.593 domicílios. A Tabela 1 mostra a distribuição da população de jovens nas 4 categorias analisadas.

Tabela 1

Distribuição dos jovens com idade de 15 a 21 anos pelas 4 categorias, no Brasil, conforme dados da PNAD de 2006

\begin{tabular}{c|c|c|c}
\hline \multirow{2}{*}{ Categoria } & \multirow{2}{*}{ № na amostra } & \multicolumn{2}{|c}{ População } \\
\cline { 3 - 4 } & & № $(1000)$ & $\%$ \\
\hline 1 - Trabalha e não estuda & 11.343 & 5.311 & 29,2 \\
\hline 2 - Trabalha e estuda & 10.265 & 4.653 & 34,2 \\
\hline 3 - Estuda e não trabalha & 14.280 & 6.215 & 11,0 \\
\hline 4 - Não trabalha nem estuda & 4.651 & 1.995 & 100,0 \\
\hline \multicolumn{1}{c|}{ Total } & 40.539 & 18.174 & \\
\hline
\end{tabular}

Verifica-se que $49,3 \%$ desses jovens são do sexo masculino e $54,8 \%$ fazem parte da PEA (população economicamente ativa). Entre os 9.964 mil que fazem parte da PEA, apenas $75,6 \%$ estão ocupados.

O valor médio de renda domiciliar per capita desses jovens é $\mathrm{R} \$ 457,30$, sendo que $82,7 \%$ desse rendimento domiciliar correspondem a rendimento de trabalho, $8,2 \%$ provêm de aposentadorias e 5,0\% corresponde a pensões.

Eliminando os rendimentos de aposentadorias e pensões e o eventual rendimento do trabalho do jovem analisado, a média desse rendimento domiciliar per capita “corrigido" é R \$359,41.

A escolaridade média dos adultos do domicílio associado a cada jovem é 7,326 anos.

Analogamente ao que fizeram Reis e Camargo (2007), pode-se iniciar a análise com um modelo de lógite binomial no qual a variável dependente é a probabilidade de pertencer à PEA (agregando as categorias 1 e 2 de um lado, e as categorias 3 e 4 de outro lado). Foi estimado um modelo com as variáveis explanatórias listadas na seção 2, considerando 4 níveis de escolaridade do jovem e uma variável binária que indica se o domicílio tem pessoa que recebe aposentadoria e/ou pensão. A estimativa do coeficiente dessa variável binária é $-0,1530$ (e estimativa do respectivo desvio padrão igual a 0,0252 ), indicando que a presença de um aposentado ou pensionista no domicílio reduz a probabilidade de o jovem participar da PEA. 
A seguir foi ajustado um modelo similar, substituindo a variável binária que indica a presença de aposentado e/ou pensionista pelo valor per capita de aposentadorias e pensões (em centenas de reais). A estimativa do coeficiente dessa variável é -0,0889 (e estimativa do respectivo desvio padrão igual a 0,0072), indicando que a renda de aposentadorias e pensões contribui de maneira estatisticamente significativa para reduzir a probabilidade de o jovem participar da PEA. Interessante constatar que nesse mesmo modelo, a estimativa do coeficiente do rendimento domiciliar per capita (também em centenas de reais) é $-0,0417$ (com estimativa do respectivo desvio padrão igual a 0,0028), mostrando que o efeito de um acréscimo na renda de aposentadorias e/ou pensões é cerca de duas vezes maior do que o efeito de um acréscimo de igual montante na renda de outras fontes.

Ressalte-se que esses modelos ajustados aos dados da PNAD de 2006 confirmam resultados obtidos por Reis e Camargo para 2003.

Havendo interesse em verificar se aposentadorias e pensões afetam a probabilidade de o jovem não trabalhar nem estudar (categoria 4), é apropriado ajustar um modelo de lógite binomial cuja variável dependente seja essa probabilidade. Para um modelo com as mesmas variáveis explanatórias do modelo anterior, a estimativa do coeficiente do rendimento de aposentadorias e pensões per capita é - 0,0137 , com desvio padrão estimado em 0,0113. O teste da hipótese de que o coeficiente é igual a zero tem probabilidade caudal igual a 22,5\%, indicando que não é razoável rejeitar a hipótese de nulidade. Um modelo igual ajustado aos dados da PNAD de 2003 levou a uma estimativa do coeficiente do rendimento de aposentadorias e/ou pensões que é negativa e estatisticamente significativa, contrariando a afirmativa de Reis e Camargo assinalada na Introdução.

A seguir são apresentados os resultados obtidos com dois modelos de lógite multinomial. No primeiro o efeito de aposentadorias e pensões sobre a probabilidade de o jovem pertencer a uma das 4 categorias descritas na seção 1 é captado por meio de uma variável binária que é igual a 1 quando o rendimento domiciliar inclui aposentadorias e/ou pensões. A Tabela 2 apresenta as estimativas dos parâmetros dessa variável e dos respectivos desvios padrões. Cabe lembrar que a categoria 1 (trabalha e não estuda) foi adotada como "base".

Tabela 2

Estimativas dos parâmetros da variável binária

que indica a presença de aposentado e/ou pensionista no domicílio do jovem. Brasil, 2006

\begin{tabular}{l|c|c}
\hline \multicolumn{1}{c|}{ Categoria } & Estimativa do parâmetro $^{(1)}$ & Estimativa do desvio padrão $^{\circ}$ \\
\hline 2 - Trabalha e estuda & 0,2944 & 0,0343 \\
\hline 3 - Estuda e não trabalha & 0,4120 & 0,0366 \\
\hline 4 - Não trabalha nem estuda & 0,1758 & 0,0419 \\
\hline
\end{tabular}

(1) Todas as estimativas são estatisticamente diferentes de zero ao nível de significância de $0,1 \%$.

O fato de a estimativa do parâmetro relativo à categoria 4 (não trabalha nem estuda) ser positivo e estatisticamente significativo não significa que a presença de um aposentado ou pensionista no domicílio aumente a probabilidade de o jovem pertencer à categoria dos que não trabalham nem estudam. 
Fixadas as demais características do jovem e do seu domicílio, pode-se usar o modelo estimado para calcular as probabilidades associadas às 4 categorias, considerando, alternativamente, valores zero ou 1 para a variável binária que indica presença de aposentado e/ou pensionista. As Tabelas 3, 4 e 5 mostram os resultados obtidos para três situações distintas. Nos três casos observa-se que a presença de aposentado e/ou pensionista no domicílio contribui para reduzir a probabilidade de "trabalhar e não estudar", aumentar substancialmente a probabilidade de "estudar e não trabalhar" e reduzir a probabilidade de "não trabalhar nem estudar".

\section{Tabela 3}

Probabilidades estimadas para as 4 categorias, na ausência ou presença de aposentado e/ou pensionista, considerando um jovem do sexo masculino, com idade média (17,982 anos), branco, com escolaridade de 1 a 8 anos, em domicílio da região MG+ES+RJ, com um outro jovem, com renda domiciliar per capita corrigida média $(\mathrm{R} \$ 359,41)$ e valor médio para escolaridade dos adultos $(7,326$ anos)

\begin{tabular}{c|c|c}
\hline \multirow{2}{*}{\multicolumn{2}{c}{ Categoria }} & \multicolumn{2}{|c}{ Probabilidades estimadas (\%) } \\
\cline { 2 - 3 } & $\begin{array}{l}\text { Sem aposentado } \\
\text { e/ou pensionista }\end{array}$ & $\begin{array}{c}\text { Com aposentado } \\
\text { e/ou pensionista }\end{array}$ \\
\hline 1 - Trabalha e não estuda & 36,5 & 29,2 \\
\hline 2 - Trabalha e estuda & 28,8 & 31,0 \\
\hline 3 - Estuda e não trabalha & 25,9 & 31,4 \\
\hline $4-$ Não trabalha nem estuda & 8,8 & 8,4 \\
\hline Total & 100,0 & 100,0 \\
\hline
\end{tabular}

Tabela 4

Probabilidades estimadas para as 4 categorias, na ausência ou presença de aposentado e/ou pensionista, considerando um jovem com 16 anos de idade, do sexo masculino, branco, com escolaridade de 1 a 8 anos, em domicílio da região MG+ES+RJ, com um outro jovem, com renda domiciliar per capita corrigida de $\mathrm{R} \$ 200,00$ e escolaridade média dos adultos igual a 2 anos

\begin{tabular}{c|c|c}
\hline \multirow{2}{*}{ Categoria } & \multicolumn{2}{|c}{ Probabilidades estimadas (\%) } \\
\cline { 2 - 3 } & $\begin{array}{l}\text { Sem aposentado } \\
\text { e/ou pensionista }\end{array}$ & $\begin{array}{l}\text { Com aposentado } \\
\text { e/ou pensionista }\end{array}$ \\
\hline 1 - Trabalha e não estuda & 20,9 & 15,7 \\
\hline 2- Trabalha e estuda & 31,7 & 32,1 \\
\hline 3- Estuda e não trabalha & 39,9 & 45,4 \\
\hline 4- Não trabalha nem estuda & 7,5 & 6,7 \\
\hline Total & 100,0 & 100,0 \\
\hline
\end{tabular}

Tabela 5

Probabilidades estimadas para as 4 categorias, na ausência ou presença de aposentado e/ou pensionista, considerando um jovem do sexo feminino e demais características iguais às da Tabela 4

\begin{tabular}{|c|c|c|}
\hline \multirow[b]{2}{*}{ Categoria } & \multicolumn{2}{|c|}{ Probabilidades estimadas $(\%)$} \\
\hline & $\begin{array}{l}\text { Sem aposentado } \\
\text { e/ou pensionista }\end{array}$ & $\begin{array}{l}\text { Com aposentado } \\
\text { e/ou pensionista }\end{array}$ \\
\hline 1 - Trabalha e não estuda & 11,9 & 8,8 \\
\hline 2 - Trabalha e estuda & 26,3 & 26,0 \\
\hline 3 - Estuda e não trabalha & 47,4 & 52,6 \\
\hline 4 - Não trabalha nem estuda & 14,4 & 12,6 \\
\hline Total & 100,0 & 100,0 \\
\hline
\end{tabular}


No outro modelo de lógite multinomial estimado, em lugar da variável binária que indica a presença de aposentado e/ou pensionista no domicílio, usa-se o valor do rendimento de aposentadorias e pensões per capita, em centenas de reais correntes. A Tabela 6 apresenta as estimativas dos parâmetros dessa variável e dos respectivos desvios padrões.

Tabela 6

Estimativas dos parâmetros da variável "rendimento de aposentadorias e pensões per capita" (em centenas de reais) do domicílio do jovem. Brasil, 2006

\begin{tabular}{l|c|c}
\multicolumn{1}{c|}{ Categoria } & Estimativa do parâmetro $^{(1)}$ & Estimativa do desvio padrão $^{\circ}$ \\
\hline 2 - Trabalha e estuda & 0,1864 & 0,0129 \\
\hline 3 - Estuda e não trabalha & 0,2458 & 0,0131 \\
\hline 4 - Não trabalha nem estuda & 0,1442 & 0,0161 \\
\hline
\end{tabular}

Novamente, o fato de a estimativa do parâmetro associado à categoria "não trabalha nem estuda" ser positivo e estatisticamente significativo não permite concluir que o rendimento de aposentadorias e pensões contribui para aumentar a probabilidade de o jovem pertencer a essa categoria.

Fixadas as demais características do jovem e do seu domicílio, pode-se usar o modelo estimado para calcular as probabilidades associadas às 4 categorias para qualquer valor do rendimento de aposentadorias e pensões. Isso foi feito para valores dessa variável de 0 a 10 (o que corresponde a valores de 0 a $\mathrm{R} \$ 1.000$ desse rendimento per capita) e, com os valores estimados das probabilidades, foi construído o Gráfico 1.

\section{Gráfico 1}

Variação das probabilidades de cada categoria de atividade do jovem em função do rendimento de aposentadorias e pensões per capita. Brasil, 2006

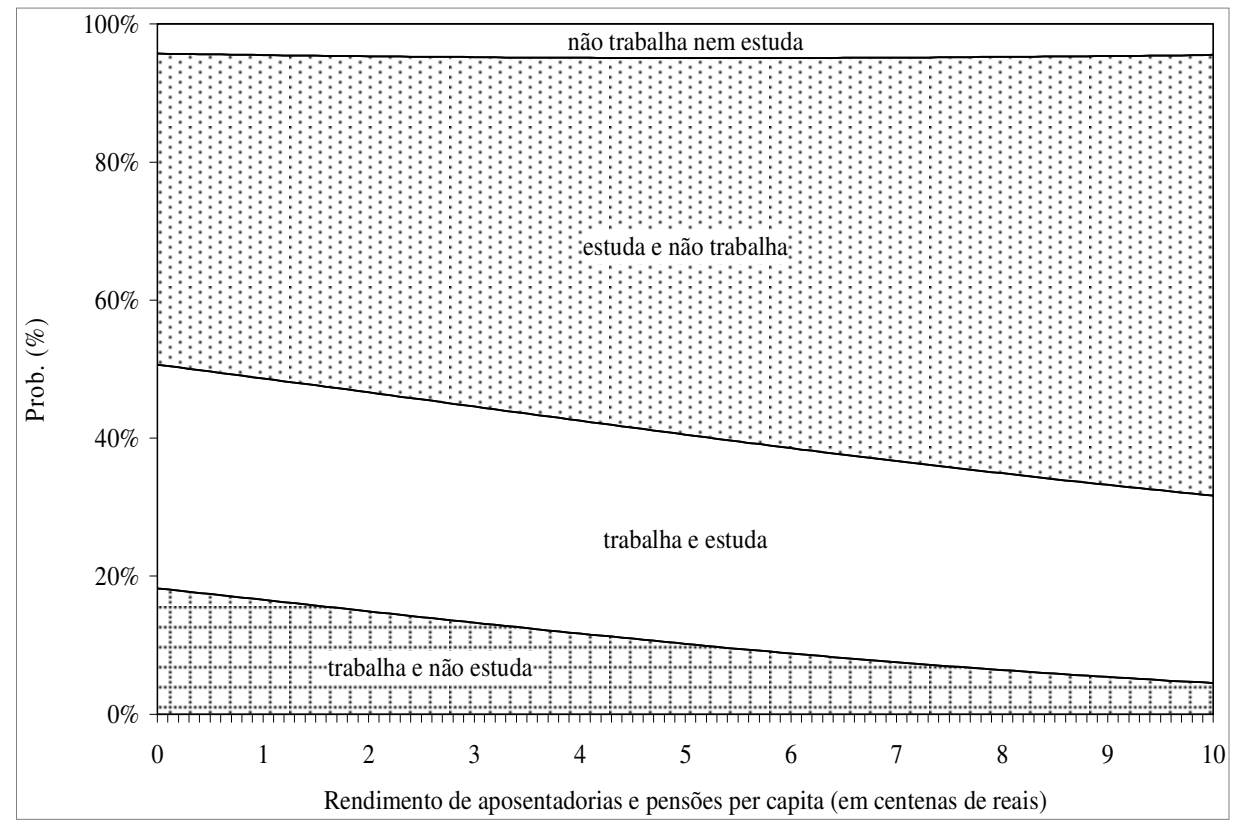


Considerou-se um jovem do sexo masculino, com idade média (17,982 anos), branco, com escolaridade de 1 a 8 anos, residente em domicílio da região MG+ES+RJ, incluindo um outro jovem, com renda domiciliar per capita corrigida média ( $\mathrm{R} \$ 359,41)$ e valor médio para escolaridade dos adultos (7,326 anos).

É notória, no Gráfico 1, a redução da probabilidade de "trabalhar e não estudar" e o crescimento da probabilidade de "estudar e não trabalhar", em função do rendimento de aposentadorias e pensões per capita.

No gráfico, a probabilidade de pertencer à categoria "não trabalha nem estuda" se mostra insensível ao valor do rendimento de aposentadorias e pensões per capita $(x)$. O exame dos valores calculados mostra que essa probabilidade estimada é $8,65 \%$ para $x=0$, sobe para $8,75 \%$ quando $x=1,5(\mathrm{R} \$ 150)$ e depois vai diminuindo, sendo igual a $6,55 \%$ quando $x=10(\mathrm{R} \$ 1.000)$.

É claro que esse tipo de análise pode ser repetido considerando outros conjuntos de características básicas do jovem e do seu domicílio. Isso foi feito para alguns casos, sem que fosse observado algum tipo de comportamento muito diferente do observado no gráfico 1.

\section{Considerações finais}

Confirmando resultados obtidos por Reis e Camargo (2007), verifica-se que o rendimento de aposentadorias e pensões contribui para reduzir a probabilidade de o jovem "trabalhar e não estudar" e para aumentar substancialmente a probabilidade de ele "estudar e não trabalhar".

A probabilidade de o jovem "não trabalhar nem estudar" é menos sensível ao nível daquele rendimento, mas os resultados obtidos indicam que a presença de aposentados e/ou pensionistas no domicílio contribui para reduzir essa probabilidade.

Como seria de se esperar, as aposentadorias e pensões têm efeitos benéficos sobre as famílias que as recebem, sendo usadas, inclusive, para investir na formação dos jovens, facilitando sua dedicação aos estudos.

É claro que cabe aprofundar a pesquisa e é bem provável que as aposentadorias e pensões, em algumas circunstâncias mais específicas, promovam uma ociosidade socialmente indesejável.

O fato de aposentadorias e pensões terem efeitos benéficos sobre as famílias que as recebem não significa, obviamente, que o sistema previdenciário no Brasil não precise de reformas, tanto para garantir a sua sustentabilidade como para eliminar as injustiças do sistema apontadas por Nicholson (2007).

\section{Referências bibliográficas}

GREENE, W. H. Econometric analysis. 4. ed. Prentice-Hall, 2000.

NICHOLSON, B. A previdência injusta: como o fim dos privilégios pode mudar o Brasil. São Paulo: Geração Editorial, 2007.

REIS, M. C.; CAMARGO, J. M. Impactos de aposentadorias e pensões sobre a educação e a participação dos jovens na força de trabalho. Pesquisa e Planejamento Econômico, Rio de Janeiro, v. 37, n. 2, ago. 2007. 